\title{
COMPORTAMENTO DE CRISTALIZAÇÃO DE LIPÍDIOS ESTRUTURADOS OBTIDOS A PARTIR DE GORDURA DE PALMISTE E ÓLEO DE PEIXE
}

\author{
Oscar Wilfredo Díaz Gamboa e Luiz Antonio Gioielli* \\ Departamento de Tecnologia Bioquímico-Farmacêutica, Faculdade de Ciências Farmacêuticas, Universidade de São Paulo, \\ Av. Prof. Lineu Prestes, 580, 05508-900 São Paulo - SP, Brasil \\ Recebido em 11/2/05; aceito em 14/10/05; publicado na web em 14/3/06
}

\begin{abstract}
CRYSTALLIZATION BEHAVIOR OF STRUCTURED LIPIDS PRODUCED FROM PALM KERNEL FAT AND FISH OIL. The aim of this study is to evaluate the crystal structure of binary mixtures of palm kernel fat and fish oil, before and after chemical and enzymatic interesterification. The crystal structure was analyzed by polarized light microscopy. The addition of fish oil didn't change the palm kernel fat crystallization characteristics, spherullites of types A and B being observed. However, due to chemical and enzymatic interesterification, smaller crystals were obtained. There was no difference between chemical and enzymatic interesterification, probably as a function of acyl migration in discontinuous processes catalyzed by lipases.
\end{abstract}

Keywords: structured lipids; interesterification; crystallization.

\section{INTRODUÇÃO}

As porcentagens relativas dos diferentes ácidos graxos na dieta humana sofreram alterações profundas em conseqüência do processamento industrial dos alimentos. O aumento de consumo de óleos vegetais e a diminuição do consumo de produtos derivados do pescado provocou aumento da relação de ácidos graxos n-6/ n-3, sendo atualmente da ordem de 20-30:1. Seria ideal que esta relação chegasse a valores de 2-4:1, típica da época em que o código genético humano foi estabelecido, em resposta à dieta ${ }^{1}$.

Tem-se verificado, nos últimos anos, que o consumo de certos óleos e gorduras tem efeitos positivos para a saúde e na redução do risco de doenças ${ }^{2,3}$. Os alimentos funcionais, dos quais os lipídios estruturados fazem parte, são alimentos ou ingredientes que podem propocionar um efeito benéfico para a saúde, além dos nutrientes básicos que eles contêm ${ }^{4}$. Os lipídios estruturados podem ser definidos como triacilgliceróis reestruturados ou modificados para alterar a composição em ácidos graxos e/ou sua distribuição nas moléculas de glicerol, por métodos químicos, enzimáticos ou de engenharia genética ${ }^{5-8}$. Embora a maioria dos lipídios estruturados seja utilizada para aplicações médicas, alguns são utilizados em alimentos, como produtos de confeitaria e chocolates ${ }^{9}$.

A obtenção e caracterização de lipídios estruturados contendo óleo de pescado e ácidos graxos poliinsaturados de cadeia longa n-3 $3^{10-21}$ têm sido realizadas principalmente por via enzimática utilizando como matérias-primas óleo de pescado refinado e triacilgliceróis purificados de óleo de pescado. Estes componentes foram interesterificados com óleos de canola e de colza, e com triacilgliceróis de cadeia média e tricaprilina. Também se utilizaram ácidos graxos livres, como os ácidos caprílico, cáprico, eicosapentaenóico, docosaexaenóico e ácidos graxos livres de óleo de girassol. Os pesquisadores obtiveram lipídios estruturados contendo de 29 a $52 \%$ de ácidos graxos poliinsaturados n-3 de cadeia longa. Estes produtos foram analisados em relação à sua composição em ácidos graxos, composição em triacilgliceróis, ácidos graxos presentes na posição sn-2, migração acil proveniente da interesterificação, estabilidade oxidativa, índices de iodo, de

*e-mail: lagio@usp.br saponificação e de peróxidos, e conteúdo de ácidos graxos livres. Contudo, estes autores não apresentaram resultados referentes às propriedades físicas destes lipídios estruturados. Por outro lado, Díaz Gamboa e Gioielli ${ }^{22,23}$ analisaram propriedades físicas de lipídios estruturados obtidos a partir de gordura de palmiste e óleo de peixe, como ponto de fusão, consistência e conteúdo de gordura sólida.

O comportamento de cristalização de lipídios tem implicações muito importantes, principalmente no processamento industrial de produtos cujas características físicas dependem em grande parte de cristais de gordura, como chocolates, margarinas e "shortenings", assim como na separação de frações específicas a partir de gorduras naturais, através do fracionamento ${ }^{24}$. As velocidades de formação e de crescimento dos cristais e as transformações polimórficas são importantes para se determinar o processo e as condições de armazenamento de óleos e gorduras ${ }^{25}$.

Como a velocidade de nucleação aumenta de forma exponencial com o aumento da supersaturação, enquanto a velocidade de crescimento é linearmente proporcional à supersaturação, o número de cristais aumenta e o tamanho dos cristais diminui quando a cristalização ocorre a baixas temperaturas. $\mathrm{O}$ resfriamento rápido a baixa temperatura, seguido de agitação intensa leva à formação de cristais pequenos, como os encontrados na margarina. Por outro lado, o resfriamento lento sob agitação suave leva à formação de cristais grandes facilmente visíveis a olho nu. Neste caso, os cristais podem formar aglomerados de esferulitos com tamanhos de centenas de micrômetros ${ }^{26,27}$.

A organização hierárquica dos níveis estruturais na rede cristalina da gordura é melhor elucidada pelo exame dos níveis estruturais formados na rede quando a gordura cristaliza a partir do estado líquido. O crescimento da rede sólida começa com sítios iniciais de nucleação, que crescem formando cristais maiores quando moléculas adicionais de triacilgliceróis cristalizam. Estes cristais maiores formam elementos microestruturais com aproximadamente o mesmo tamanho $(<10 \mu \mathrm{m})$, que então se agregam em grupos maiores, chamados de microestruturas $(>100 \mu \mathrm{m})$. Estes grupos se empacotam de modo regular e homogêneo, formando os blocos estruturais da rede cristalina da gordura. Entre os elementos microestruturais e as microestruturas fica a fase líquida da rede (óleo $)^{28,29}$. Quando a 
rede cristalina é submetida a uma tensão, as ligações entre as microestruturas têm maior probabilidade de serem rompidas que os elementos microestruturais dentro delas. Portanto, as ligações mais fracas são aquelas entre as microestruturas. Esta situação é chamada apropriadamente, de teoria dos elos fracos ${ }^{30}$.

Um aspecto fundamental nas técnicas de processamento de gorduras é sua tendência à cristalização. As gorduras são polimórficas, podendo existir em três principais formas cristalinas, $\alpha, \beta$-prima e $\beta$. A forma $\beta$ é mais densamente empacotada e mais estável, o que implica em maiores consistência e ponto de fusão ${ }^{27}$.

A característica desejável de cristalização de margarinas é na forma $\beta$-prima, como ocorre na manteiga. Contudo, é possível obter produtos adequados mesmo usando gorduras com alta propensão à forma $\beta$, como o óleo de soja totalmente hidrogenado. A formação de isômeros trans na hidrogenação parcial de óleos influencia a cristalização, favorecendo a formação de formas $\beta$-prima ${ }^{26,31}$.

A textura de produtos gordurosos depende das propriedades macroscópicas da gordura. Dentre essas propriedades, pode-se citar a espalhabilidade de margarinas e manteigas. As propriedades macroscópicas são influenciadas particularmente pela microestrutura das gorduras. Por isso, é importante considerar os efeitos da microestrutura para posterior análise das propriedades macroscópicas $^{32}$.

O presente trabalho teve o objetivo de avaliar a estrutura cristalina das misturas binárias de óleo de peixe e gordura de palmiste, bem como de seus correspondentes lipídios estruturados obtidos por vias química e enzimática.

\section{PARTE EXPERIMENTAL}

\section{Material}

Foram utilizados como matérias-primas óleo refinado de peixe e gordura refinada de palmiste. O óleo de peixe (OmegaVit EPA) foi fornecido gentilmente pela empresa BASF. É óleo natural de peixes, refinado, desodorizado e estabilizado com tocoferol. Contém EPA (17\%), DHA (11\%), outros n-3 PUFAs (5\%), outros PUFAs (12\%) e MUFAs (24\%), segundo especificação do fabricante. A gordura refinada de palmiste foi fornecida gentilmente pela empresa Agropalma S.A.

\section{Mistura}

Para o estudo das interações em misturas binárias de gorduras realizou-se um planejamento de 6 experimentos (Tabela 1). A gordura de palmiste e o óleo de peixe são representados por $\mathrm{x}_{1}$ e $\mathrm{x}_{2}$, respectivamente, sendo que $x_{1}+x_{2}=1$ ou $100 \%$. Duas amostras representam os componentes isoladamente e quatro são formadas por misturas binárias. As amostras foram preparadas por mistura nas proporções mencionadas, após fusão completa à temperatura de $60-70{ }^{\circ} \mathrm{C}$ e novamente solidificadas, sendo armazenadas sob resfriamento.

Tabela 1. Planejamento experimental das misturas

\begin{tabular}{lcc}
\hline Amostra $\left(\mathrm{n}^{\circ}\right)$ & \multicolumn{2}{c}{ Proporções $(\mathrm{p} / \mathrm{p})$} \\
\cline { 2 - 3 } & $\mathrm{x}_{1}-$ gordura de palmiste & $\mathrm{x}_{2}$ - óleo de peixe \\
\hline 1 & 100 & 0 \\
2 & 80 & 20 \\
3 & 60 & 40 \\
4 & 40 & 60 \\
5 & 20 & 80 \\
6 & 0 & 100 \\
\hline
\end{tabular}

\section{Composição em ácidos graxos}

A análise da composição em ácidos graxos foi realizada em cromatógrafo à gás Varian GC, modelo $3400 \mathrm{CX}$, equipado com detector de ionização de chama e "Workstation Star Chromatography". Foi utilizada coluna capilar de sílica fundida CP WAX 52CB (Chrompack), com $30 \mathrm{~m}$ de comprimento x 0,25 mm de diâmetro interno e contendo $0,25 \mu \mathrm{m}$ de polietilenoglicol. As condições foram: injeção split, razão de $50: 1$; temperatura da coluna: $150^{\circ} \mathrm{C}$ por $5 \mathrm{~min}$, programada até $215^{\circ} \mathrm{C}$ em uma razão de $3{ }^{\circ} \mathrm{C}$ por minuto; gás de arraste: hélio, em uma vazão de $1,5 \mathrm{~mL} / \mathrm{min}$; gás "make-up": hélio a $30 \mathrm{~mL} / \mathrm{min}$; temperatura do injetor: $250{ }^{\circ} \mathrm{C}$; temperatura do detector: $280{ }^{\circ} \mathrm{C}$. A composição qualitativa foi determinada por comparação dos tempos de retenção dos picos com os dos respectivos padrões de ácidos graxos (Sigma 189-1, 189-3, 189-6, 189-19 e Supelco 1895-1, 1-8915-1). A composição quantitativa foi realizada por normalização de área, sendo expressa como porcentagem em massa ${ }^{33}$.

\section{Interesterificação}

A interesterificação química foi catalisada por metóxido de sódio. Este foi preparado pela evaporação do metanol de uma solução comercial de metilato de sódio a $30 \%$, na véspera de cada reação. O metóxido de sódio seco foi triturado em graal de vidro. As amostras a serem rearranjadas foram previamente fundidas em forno de microondas até completa dissolução dos cristais. A seguir, $320 \mathrm{~g}$ de cada amostra foram aquecidas durante $1 \mathrm{~h}$ em evaporador rotativo, em balão de $500 \mathrm{~mL}$, utilizando-se banho de água à temperatura entre $90-95{ }^{\circ} \mathrm{C}$, sob pressão reduzida (56 mm de $\mathrm{Hg}$ ) proporcionada por bomba de vácuo, para remoção de traços de umidade do material. Após esta etapa, o sistema foi resfriado a $60{ }^{\circ} \mathrm{C}$. A reação de interesterificação foi realizada em balão de três bocas de $500 \mathrm{~mL}$ imerso em banho de água à temperatura de $60{ }^{\circ} \mathrm{C}$, sendo a agitação proporcionada por barra magnética e mantendo-se a pressão reduzida. $\mathrm{O}$ catalisador foi usado na proporção de $0,4 \%$. Para facilitar a dispersão do catalisador, este foi pré-misturado a uma porção da gordura a ser rearranjada, sendo então adicionado ao restante, sob agitação. Imediatamente antes da adição do catalisador foi retirada uma amostra inicial, correspondendo como controle. Após 60 min de reação foi adicionada água destilada para inativar o catalisador, mantendo-se a agitação por mais $3 \mathrm{~min}$. A gordura rearranjada foi então filtrada a quente, sob pressão reduzida, sobre sulfato de sódio e kieselgur. A filtração teve por finalidade a retenção de umidade e a remoção de sabões e compostos escuros forma$\operatorname{dos}^{22,33-35}$.

A interesterificação enzimática foi catalisada pela enzima imobilizada sn-1,3 específica de Rhizomucor miehei (Novozymes). As amostras de gordura a serem interesterificadas foram previamente fundidas em forno de microondas até completa dissolução dos cristais. A seguir, $160 \mathrm{~g}$ de cada amostra foram colocadas em balão de três bocas de $250 \mathrm{~mL}$. Antes da adição da enzima, foi adicionada às amostras água destilada na proporção de $0,3 \%$. A enzima foi utilizada na proporção de $5 \%$. A reação foi realizada em banho de água à temperatura de $65^{\circ} \mathrm{C}$ por período de $6 \mathrm{~h}$, sob agitação proporcionada por barra magnética e mantendo o sistema em atmosfera de nitrogênio. Após a reação, as partículas do catalisador foram removidas por filtração $23,36,37$.

\section{Microscopia sob luz polarizada}

As amostras foram fundidas à temperatura de $50{ }^{\circ} \mathrm{C}$ em forno de microondas e, com a ajuda de um tubo capilar, colocou-se uma gota de amostra sobre uma lâmina de vidro pré-aquecida em tem- 
peratura controlada $\left(50{ }^{\circ} \mathrm{C}\right)$ e cobriu-se com uma lamínula. As lâminas foram mantidas à temperatura de análise $\left(10,15\right.$ e $\left.20{ }^{\circ} \mathrm{C}\right)$ durante $5 \mathrm{~h}^{38,39}$.

O microscópio de luz polarizada (Olympus, modelo BX 50) é ligado a uma câmera de vídeo digital (Media Cybernetics). As lâminas foram colocadas sobre o suporte da placa de aquecimento, mantida à mesma temperatura de cristalização. As imagens foram capturadas pelo aplicativo Image Pro-Plus versão 4.5.1.22 (Media Cybernetics), utilizando luz polarizada e com ampliação de 40 vezes. Para cada lâmina foi escolhido um campo representativo. Foram determinados os diâmetros dos cristais e dos aglomerados ${ }^{39-41}$.

\section{RESULTADOS E DISCUSSÃO}

A Tabela 2 mostra a composição em ácidos graxos da gordura de palmiste, do óleo de peixe e das respectivas misturas binárias. Os resultados indicam que as composições da gordura de palmiste e do óleo de peixe estão de acordo com os limites citados na literatura e dentro das especificações comerciais dos fabricantes ${ }^{42,43}$. A gordura de palmiste apresentou 79,8\% de ácidos graxos saturados, principalmente de cadeia média, ao passo que o óleo de peixe mostrou $65,0 \%$ de ácidos graxos poliinsaturados, principalmente de cadeia longa.
Os resultados apresentados nas Tabelas 3 e 4 mostram que a composição em ácidos graxos dos lipídios estruturados é muito semelhante àquela apresentada na Tabela 2 , referente às gorduras originais e às misturas. Isto confirma que a interesterificação, seja química ou enzimática, não promove alteração na composição em ácidos graxos das amostras. As diferenças obtidas podem ser atribuídas a variações decorrentes dos métodos experimentais empregados. Obtiveram-se lipídios estruturados contendo de 5,0 a 23,4\% de ácidos graxos poliinsaturados n-3 de cadeia longa. Estes teores estiveram abaixo das porcentagens obtidas por outros autores, na faixa de 29 a $52 \%{ }^{10-21}$. Isto se deve ao fato que, neste trabalho, se procurou obter lipídos estruturados contendo ácidos graxos n-3 e que apresentassem características plásticas, que aumentariam suas possibilidades de aplicação prática. Isto fez com que os teores dos ácidos graxos n-3 não pudessem ser muito elevados. Os trabalhos relatados na literatura, com maiores teores de ácidos graxos n-3, levam à formação de lipídios estruturados que se apresentam no estado líquido à temperatura ambiente.

É importante obter lipídios estruturados que apresentem características físicas provenientes do estado sólido, pois a demanda para óleos líquidos é somente $1 / 3$ da produção total de óleos e gorduras, enquanto que $2 / 3$ dos óleos são consumidos no estado semi-sólido, após algum tipo de modificação. Isto ocorre porque,

Tabela 2. Composição em ácidos graxos da gordura de palmiste, do óleo de peixe e suas misturas binárias

\begin{tabular}{|c|c|c|c|c|c|c|}
\hline \multirow{2}{*}{$\begin{array}{l}\text { Ácido graxo } \\
(\%)\end{array}$} & \multicolumn{6}{|c|}{$\operatorname{Amostra}\left(\mathrm{n}^{\circ}\right)^{*}$} \\
\hline & $1\left(\mathrm{x}_{1}\right)(100: 0)$ & 2(80: 20) & $3(60: 40)$ & 4(40: 60$)$ & 5(20: 80) & $6\left(\mathrm{x}_{2}\right)(0: 100)$ \\
\hline $6: 0$ & 0,20 & 0,24 & - & - & - & - \\
\hline 8:0 & 3,13 & 3,19 & 3,20 & 1,45 & 0,71 & - \\
\hline $10: 0$ & 3,18 & 3,10 & 2,97 & 1,43 & 0,71 & - \\
\hline $12: 0$ & 45,51 & 42,00 & 37,58 & 20,00 & 10,24 & 0,17 \\
\hline $14: 0$ & 15,64 & 14,68 & 14,01 & 11,20 & 9,51 & 9,02 \\
\hline $15: 0$ & - & - & - & 0,30 & 0,39 & 0,56 \\
\hline $16: 0$ & 9,48 & 10,10 & 11,18 & 13,80 & 14,77 & 17,44 \\
\hline $16: 1$ & 0,09 & 1,64 & 3,23 & 5,35 & 7,13 & 9,53 \\
\hline $16: 2 n-7$ & - & 0,21 & 0,44 & 0,71 & 0,95 & 1,30 \\
\hline $16: 2 n-4$ & - & - & - & 0,19 & 0,40 & 0,51 \\
\hline 17:0 & - & - & - & 0,26 & 0,34 & 0,44 \\
\hline $17: 1$ & - & 2,27 & 0,54 & 1,01 & 1,35 & 1,90 \\
\hline $16: 4$ & - & 0,38 & 0,80 & 1,26 & 1,76 & 2,28 \\
\hline $18: 0$ & 2,55 & 2,36 & 2,26 & 2,92 & 3,02 & 3,20 \\
\hline $18: 1 n-9$ & 16,90 & 13,74 & 12,50 & 14,40 & 13,59 & 13,10 \\
\hline $18: 2 n-6$ & 2,64 & 2,14 & 1,57 & 1,71 & 1,44 & 1,11 \\
\hline $18: 2 n-4$ & - & - & - & 0,24 & 0,31 & 0,40 \\
\hline $18: 3 n-6$ & - & - & - & 0,15 & 0,20 & 0,26 \\
\hline $18: 3 n-3$ & 0,13 & - & - & 0,37 & 0,50 & 0,64 \\
\hline $18: 4 n-3$ & - & 0,39 & 0,76 & 1,37 & 1,80 & 2,30 \\
\hline 20:0 & 0,13 & - & - & 0,24 & 0,28 & 0,30 \\
\hline $20: 1$ & 0,13 & 0,21 & - & 0,57 & 0,73 & 0,85 \\
\hline $20: 2$ & - & - & - & - & 0,20 & 0,20 \\
\hline $20: 3$ & - & - & - & - & 0,13 & - \\
\hline $20: 4 n-6$ & - & 0,16 & - & 0,57 & 0,76 & 0,92 \\
\hline $20: 5 n-3$ & - & 2,90 & 5,36 & 10,50 & 14,44 & 17,00 \\
\hline $22: 0$ & - & - & - & 0,39 & 0,13 & 0,62 \\
\hline $22: 1 n-11$ & - & - & - & - & 0,60 & - \\
\hline $22: 2$ & - & - & - & 0,42 & 0,64 & 0,64 \\
\hline $22: 5 n-3$ & - & 0,35 & 0,61 & 1,30 & 1,75 & 2,05 \\
\hline $22: 6 n-3$ & - & 1,61 & 2,99 & 6,30 & 8,40 & 10,00 \\
\hline ni & 0,29 & 0,33 & 0,00 & 1,59 & 2,85 & 3,26 \\
\hline
\end{tabular}

* Média de três determinações; $\mathrm{x}_{1}=$ gordura de palmiste; $\mathrm{x}_{2}=$ óleo de peixe $\mathrm{e}, \mathrm{ni}=$ não identificado 
Tabela 3. Composição em ácidos graxos dos lipídios estruturados por interesterificação química das misturas binárias de gordura de palmiste e óleo de peixe

\begin{tabular}{|c|c|c|c|c|}
\hline \multirow{2}{*}{$\begin{array}{l}\text { Ácido graxo } \\
(\%)\end{array}$} & \multicolumn{4}{|c|}{ Amostra $\left(n^{o}\right)^{*}$} \\
\hline & $2(80: 20)$ & $3(60: 40)$ & $4(40: 60)$ & $5(20: 80)$ \\
\hline $6: 0$ & - & - & - & - \\
\hline $8: 0$ & 3,04 & 2,18 & 1,67 & 0,76 \\
\hline 10:0 & 2,89 & 2,11 & 1,55 & 0,69 \\
\hline $12: 0$ & 0,62 & 29,60 & 21,31 & 10,02 \\
\hline $14: 0$ & 15,01 & 13,09 & 11,65 & 9,69 \\
\hline $15: 0$ & - & 0,21 & 0,31 & 0,41 \\
\hline $16: 0$ & 10,72 & 12,38 & 13,80 & 15,83 \\
\hline ni & - & - & 0,16 & 0,20 \\
\hline $16: 1$ & 1,77 & 3,58 & 5,40 & 7,26 \\
\hline ni & - & 0,61 & 1,09 & 1,83 \\
\hline $17: 0$ & - & 0,19 & 0,26 & 0,36 \\
\hline $17: 1$ & - & 0,59 & 0,84 & 1,12 \\
\hline $16: 4$ & 0,38 & 0,79 & 1,30 & 1,60 \\
\hline 18:0 & 2,60 & 2,82 & 2,92 & 3,19 \\
\hline $18: 1 n-9$ & 14,91 & 14,09 & 12,70 & 11,78 \\
\hline ni & 0,63 & 1,25 & 1,94 & 2,68 \\
\hline $18: 2 n-6$ & 2,32 & 2,08 & 1,73 & 1,46 \\
\hline ni & - & - & 0,23 & 0,32 \\
\hline $18: 3 n-6$ & 0,14 & 0,14 & 0,15 & 0,21 \\
\hline ni & - & - & - & 0,14 \\
\hline $18: 3 n-3$ & 0,24 & 0,24 & 0,35 & 0,51 \\
\hline $18: 4 n-3$ & - & 00,86 & 1,26 & 1,72 \\
\hline $\mathrm{n}: \mathrm{i}$ & - & - & 0,11 & 0,16 \\
\hline $20: 0$ & - & 0,20 & 0,24 & 0,30 \\
\hline $20: 1$ & - & 0,42 & 0,59 & 0,80 \\
\hline ni & - & - & 0,16 & 0,39 \\
\hline $20: 2$ & - & - & - & 0,12 \\
\hline $20: 3$ & - & 0,36 & 0,60 & 0,77 \\
\hline $20: 4 n-6$ & - & - & - & 0,56 \\
\hline $20: 5 n-3$ & 2,97 & 6,60 & 9,50 & 13,61 \\
\hline $22: 0$ & - & 0,27 & 0,38 & 0,56 \\
\hline $22: 1 n-11$ & - & - & - & 0,14 \\
\hline $22: 2$ & - & 0,25 & 0,36 & 0,53 \\
\hline ni & - & 0,12 & 0,16 & 0,27 \\
\hline $22: 5 n-3$ & 0,38 & 0,72 & 1,20 & 1,77 \\
\hline $22: 6 n-3$ & 1,64 & 3,80 & 5,31 & 8,01 \\
\hline ni & - & 0,16 & 0,21 & 0,23 \\
\hline
\end{tabular}

* Média de duas determinações e ni = não identificado

com exceção dos óleos para salada e fritura, todos os produtos gordurosos são submetidos a alguma forma de cristalização antes da embalagem $^{44}$.

As Tabelas 5 a 10 apresentam os diâmetros dos cristais individuais e dos aglomerados das amostras, às temperaturas de $10,15 \mathrm{e}$ $20{ }^{\circ} \mathrm{C}$, antes e após as reações de interesterificação química e enzimática.

As Figuras 1 a 6 apresentam a estrutura cristalina das amostras antes e após a interesterificação química e enzimática, por cristalização lenta na faixa de temperatura de 10 a $20^{\circ} \mathrm{C}$. Os cristais obser-

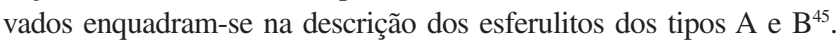

As técnicas de análise microscópica são freqüentemente as mais apropriadas para avaliar a estrutura de alimentos, visto que fornecem resultados tanto na forma de imagens como em dados numéricos. O sucesso nas medidas exige vários estágios, incluindo obter uma imagem representativa do material, analisar a imagem ade-
Tabela 4. Composição em ácidos graxos dos lipídios estruturados por interesterificação enzimática das misturas binárias de gordura de palmiste e óleo de peixe

\begin{tabular}{|c|c|c|c|c|}
\hline \multirow{2}{*}{$\begin{array}{l}\text { Ácido graxo } \\
(\%)\end{array}$} & \multicolumn{4}{|c|}{$\operatorname{Amostra}\left(n^{o}\right)^{*}$} \\
\hline & $2(80: 20)$ & $3(60: 40)$ & $4(40: 60)$ & $5(20: 80)$ \\
\hline $6: 0$ & - & - & - & - \\
\hline $8: 0$ & 3,11 & 2,24 & 1,81 & 1,09 \\
\hline 10:0 & 2,90 & 2,01 & 1,72 & 1,03 \\
\hline $12: 0$ & 40,94 & 30,48 & 22,64 & 14,04 \\
\hline $14: 0$ & 14,59 & 12,98 & 11,80 & 11,95 \\
\hline $15: 0$ & - & 0,20 & 0,31 & 0,47 \\
\hline $16: 0$ & 10,26 & 12,20 & 13,52 & 16,32 \\
\hline ni & - & 0,15 & 0,15 & 0,22 \\
\hline $16: 1$ & 1,36 & 3,45 & 5,35 & 7,82 \\
\hline ni & 0,17 & 0,23 & 1,21 & 1,82 \\
\hline $17: 0$ & 0,09 & 0,18 & 0,26 & 0,35 \\
\hline $17: 1$ & 0,21 & 0,53 & 0,12 & 1,25 \\
\hline $16: 4$ & 0,30 & 0,74 & 0,85 & 1,75 \\
\hline 18:0 & 2,46 & 2,84 & 2,72 & 2,91 \\
\hline $18: 1 n-9$ & 14,87 & 13,60 & 11,95 & 10,76 \\
\hline ni & 0,47 & 1,19 & 1,83 & 2,42 \\
\hline $18: 2 n-6$ & 2,41 & 1,97 & 1,70 & 1,35 \\
\hline ni & - & - & 0,22 & 0,29 \\
\hline $18: 3 n-6$ & 0,12 & 0,15 & 00,15 & 00,20 \\
\hline ni & - & - & - & - \\
\hline $18: 3 n-3$ & 0,09 & 0,23 & 0,36 & 0,44 \\
\hline $18: 4 n-3$ & 0,33 & 0,82 & 1,27 & 1,67 \\
\hline $\mathrm{n}: \mathrm{i}$ & - & - & 0,11 & - \\
\hline 20:0 & 0,14 & 0,19 & 0,21 & 0,23 \\
\hline $20: 1$ & 0,22 & 0,41 & 0,52 & 0,62 \\
\hline ni & - & 0,10 & 0,15 & - \\
\hline $20: 2$ & - & - & - & - \\
\hline $20: 3$ & - & - & 0,36 & - \\
\hline $20: 4 n-6$ & 0,13 & 0,35 & 0,49 & 0,62 \\
\hline ni & 0,12 & 0,28 & 00,40 & 00,49 \\
\hline $20: 5 n-3$ & 2,58 & 6,41 & 9,38 & 11,35 \\
\hline 22:0 & 0,09 & 0,26 & 0,33 & 0,41 \\
\hline $22: 1 n-11$ & - & - & - & 1,31 \\
\hline $22: 2$ & 0,09 & 0,25 & 0,35 & 0,40 \\
\hline ni & - & - & - & - \\
\hline $22: 5 n-3$ & 0,33 & 0,83 & 1,13 & 1,25 \\
\hline $22: 6 n-3$ & 1,45 & 4,07 & 5,27 & 6,00 \\
\hline ni & - & 0,15 & 0,17 & 0,21 \\
\hline
\end{tabular}

* Média de duas determinacões e, ni = não identificado

quadamente e interpretar os dados resultantes ${ }^{46}$.

Quando a temperatura de cristalização está distante do ponto de fusão da amostra, ocorre formação de cristais menores e em maior número. Próximo ao ponto de fusão, a amostra apresenta pequeno conteúdo de gordura sólida e os cristais formados são pequenos e não possuem forma distinta, ou mesmo não se formam. Deste modo, uma temperatura intermediária de cristalização auxilia na formação de cristais maiores, em menor número e com formas distintas.

Nas Tabelas 5 a 10 os altos valores do desvio-padrão em relação à média do diâmetro, ou seja, altos coeficientes de variação, são característicos de gorduras cristalizadas quando observadas sob luz polarizada ${ }^{48,49}$.

A Figura 1A mostra as imagens digitalizadas da estrutura cristalina da amostra 1 (gordura de palmiste) antes da interesterificação. As amostras apresentaram densa rede formada por cristais peque- 
Tabela 5. Diâmetros dos cristais individuais e dos aglomerados da amostra 1 (gordura de palmiste), às temperaturas de 10,15 e 20 ${ }^{\circ} \mathrm{C}$, antes e após a interesterificação química e enzimática

\begin{tabular}{lcc}
\hline $\begin{array}{l}\text { Temperatura } \\
\left({ }^{\circ} \mathrm{C}\right)\end{array}$ & $\begin{array}{c}\text { Diâmetro } \\
(\mu \mathrm{m} \pm \mathrm{dp})\end{array}$ & $\begin{array}{c}\text { Aglomerado } \\
(\mu \mathrm{m} \pm \mathrm{dp})\end{array}$ \\
\hline $10-\mathrm{NI}$ & $4,97 \pm 2,21$ & - \\
$10-\mathrm{IQ}$ & $4,96 \pm 2,23$ & - \\
$10-\mathrm{IE}$ & $4,74 \pm 2,12$ & - \\
$15-\mathrm{NI}$ & $4,94 \pm 2,19$ & - \\
$15-\mathrm{IQ}$ & $4,88 \pm 2,17$ & - \\
$15-\mathrm{IE}$ & $4,72 \pm 2,12$ & - \\
& & - \\
$20-\mathrm{NI}$ & $212,14 \pm 24,75$ & - \\
$20-\mathrm{IQ}$ & $148,09 \pm 10,24$ & \\
$20-\mathrm{IE}$ & $216,31 \pm 35,63$ & \\
\hline NI $=$ não interesterificada; IQ $=$ interesterificação química e, IE $=$ \\
interesterificação enzimática
\end{tabular}

Tabela 6. Diâmetros dos cristais individuais e dos aglomerados da amostra 2 (gordura de palmiste/óleo de peixe na proporção 80:20), às temperaturas de 10,15 e $20^{\circ} \mathrm{C}$, antes e após a interesterificação química e enzimática

\begin{tabular}{lcc}
\hline $\begin{array}{l}\text { Temperatura } \\
\left({ }^{\circ} \mathrm{C}\right)\end{array}$ & $\begin{array}{c}\text { Diâmetro } \\
(\mu \mathrm{m} \pm \mathrm{dp})\end{array}$ & $\begin{array}{c}\text { Aglomerado } \\
(\mu \mathrm{m} \pm \mathrm{dp})\end{array}$ \\
\hline $10-\mathrm{NI}$ & $4,74 \pm 2,13$ & $820,36 \pm 150,56$ \\
$10-\mathrm{IQ}$ & $4,96 \pm 2,21$ & $201,70 \pm 28,29$ \\
$10-\mathrm{IE}$ & $4,93 \pm 2,19$ & $685,12 \pm 26,45$ \\
& & \\
$15-\mathrm{NI}$ & $4,97 \pm 2,19$ & $190,65 \pm 39,71$ \\
$15-\mathrm{IQ}$ & $4,93 \pm 2,18$ & $171,02 \pm 29,54$ \\
$15-\mathrm{IE}$ & $121,65 \pm 20,09$ & $598,09 \pm 62,55$ \\
& & - \\
$20-\mathrm{NI}$ & $402,53 \pm 84,52$ & - \\
$20-\mathrm{IQ}$ & $136,66 \pm 43,75$ & - \\
$20-\mathrm{IE}$ & $189,65 \pm 52,49$ & \\
\hline
\end{tabular}

$\mathrm{NI}=$ não interesterificada; IQ = interesterificação química e, IE = interesterificação enzimática

Tabela 7. Diâmetros dos cristais individuais e dos aglomerados da amostra 3 (gordura de palmiste/óleo de peixe na proporção 60:40), às temperaturas de 10,15 e $20^{\circ} \mathrm{C}$, antes e após a interesterificação química e enzimática

\begin{tabular}{lcc}
\hline $\begin{array}{l}\text { Temperatura } \\
\left({ }^{\circ} \mathrm{C}\right)\end{array}$ & $\begin{array}{c}\text { Diâmetro } \\
(\mu \mathrm{m} \pm \mathrm{dp})\end{array}$ & $\begin{array}{c}\text { Aglomerado } \\
(\mu \mathrm{m} \pm \mathrm{dp})\end{array}$ \\
\hline $10-\mathrm{NI}$ & $4,69 \pm 2,13$ & $221,71 \pm 26,89$ \\
$10-\mathrm{IQ}$ & $4,91 \pm 2,19$ & $188,53 \pm 51,34$ \\
$10-\mathrm{IE}$ & $4,92 \pm 2,18$ & $94,97 \pm 10,96$ \\
& & - \\
$15-\mathrm{NI}$ & $435,70 \pm 95,79$ & $263,99 \pm 40,51$ \\
$15-\mathrm{IQ}$ & $4,79 \pm 2,14$ & $536,40 \pm 40,32$ \\
$15-\mathrm{IE}$ & $24,57 \pm 14,93$ & - \\
& - & - \\
$20-\mathrm{NI}$ & $146,60 \pm 27,02$ & - \\
$20-\mathrm{IQ}$ & $183,88 \pm 26,31$ & $\mathrm{IE}$
\end{tabular}

$\mathrm{NI}=$ não interesterificada; IQ = interesterificação química e, IE = interesterificação enzimática
Tabela 8. Diâmetros dos cristais individuais e dos aglomerados da amostra 4 (gordura de palmiste/óleo de peixe na proporção 40:60), às temperaturas de 10,15 e $20^{\circ} \mathrm{C}$, antes e após a interesterificação química e enzimática

\begin{tabular}{lcc}
\hline $\begin{array}{l}\text { Temperatura } \\
\left({ }^{\circ} \mathrm{C}\right)\end{array}$ & $\begin{array}{c}\text { Diâmetro } \\
(\mu \mathrm{m} \pm \mathrm{dp})\end{array}$ & $\begin{array}{c}\text { Aglomerado } \\
(\mu \mathrm{m} \pm \mathrm{dp})\end{array}$ \\
\hline $10-\mathrm{NI}$ & $4,51 \pm 2,06$ & $235,12 \pm 61,30$ \\
$10-\mathrm{IQ}$ & $43,00 \pm 18,93$ & - \\
$10-\mathrm{IE}$ & $19,56 \pm 8,14$ & - \\
& & - \\
$15-\mathrm{NI}$ & $372,28 \pm 107,70$ & - \\
$15-\mathrm{IQ}$ & $144,68 \pm 14,65$ & - \\
$15-\mathrm{IE}$ & $17,46 \pm 7,66$ & - \\
& - & - \\
$20-\mathrm{NI}$ & $-\mathrm{IQ}$ & - \\
$20-\mathrm{IE}$ & $95,29 \pm 25,47$ & - \\
\hline
\end{tabular}

NI = não interesterificada; IQ = interesterificação química e, IE = interesterificação enzimática

Tabela 9. Diâmetros dos cristais individuais e dos aglomerados da amostra 5 (gordura de palmiste/óleo de peixe na proporção de 20:80), às temperaturas de 10,15 e $20{ }^{\circ} \mathrm{C}$, antes e após a interesterificação química e enzimática

\begin{tabular}{lcc}
\hline $\begin{array}{l}\text { Temperatura } \\
\left({ }^{\circ} \mathrm{C}\right)\end{array}$ & $\begin{array}{c}\text { Diâmetro } \\
(\mu \mathrm{m} \pm \mathrm{dp})\end{array}$ & $\begin{array}{c}\text { Aglomerado } \\
(\mu \mathrm{m} \pm \mathrm{dp})\end{array}$ \\
\hline $10-\mathrm{NI}$ & $386,01 \pm 108,28$ & - \\
$10-\mathrm{IQ}$ & $29,85 \pm 17,94$ & - \\
$10-\mathrm{IE}$ & $9,61 \pm 3,49$ & - \\
& - & - \\
$15-\mathrm{NI}$ & $25,62 \pm 15,06$ & - \\
$15-\mathrm{IQ}$ & $9,33 \pm 3,53$ & - \\
$15-\mathrm{IE}$ & - & - \\
$20-\mathrm{NI}$ & $99,77 \pm 22,83$ & - \\
$20-\mathrm{IQ}$ & $47,51 \pm 19,28$ & - \\
$20-\mathrm{IE}$ &
\end{tabular}

$\mathrm{NI}=$ não interesterificada; IQ = interesterificação química e, IE = interesterificação enzimática

Tabela 10. Diâmetros dos cristais individuais e dos aglomerados da amostra 6 (óleo de peixe), às temperaturas de 10,15 e $20{ }^{\circ} \mathrm{C}$, antes e após a interesterificação química e enzimática

\begin{tabular}{lcc}
\hline $\begin{array}{l}\text { Temperatura } \\
\left({ }^{\circ} \mathrm{C}\right)\end{array}$ & $\begin{array}{c}\text { Diâmetro } \\
(\mu \mathrm{m} \pm \mathrm{dp})\end{array}$ & $\begin{array}{c}\text { Aglomerado } \\
(\mu \mathrm{m} \pm \mathrm{dp})\end{array}$ \\
\hline $10-\mathrm{NI}$ & - & - \\
$10-\mathrm{IQ}$ & $10,41 \pm 3,38$ & - \\
$10-\mathrm{IE}$ & $10,48 \pm 4,64$ & - \\
& - & - \\
$15-\mathrm{NI}$ & $25,53 \pm 12,36$ & - \\
$15-\mathrm{IQ}$ & $9,52 \pm 3,52$ & - \\
$15-\mathrm{IE}$ & - & - \\
$20-\mathrm{NI}$ & $181,33 \pm 39,11$ & - \\
$20-\mathrm{IQ}$ & $9,01 \pm 3,73$ & - \\
$20-\mathrm{IE}$ &
\end{tabular}

$\mathrm{NI}=$ não interesterificada; IQ = interesterificação química e, IE = interesterificação enzimática 

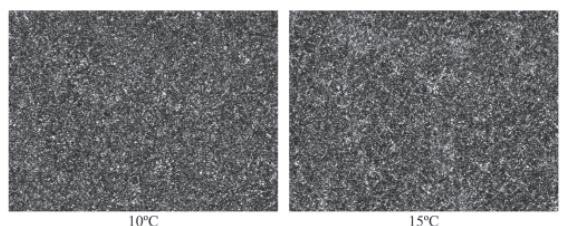

A. Não interesterificada
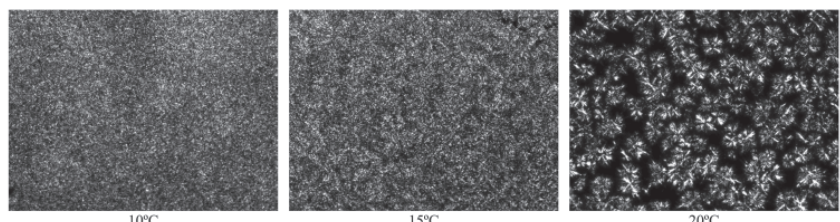

B. Interesterificada quimicamente
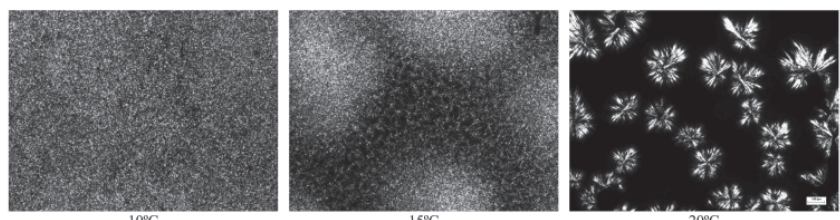

C. Interesterificada enzimaticamente

Figura 1. Imagens digitais dos cristais da amostra 1 (gordura de palmiste), em função da temperatura (a barra representa $100 \mu \mathrm{m}$ )
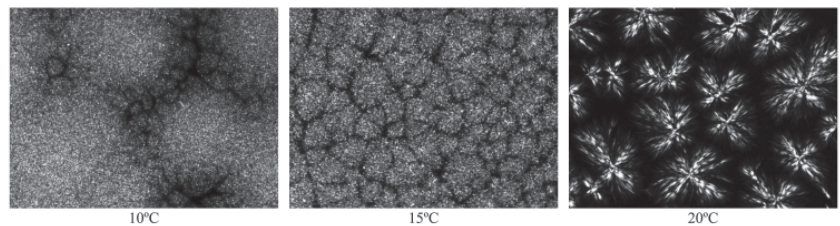

A. Não interesterificada
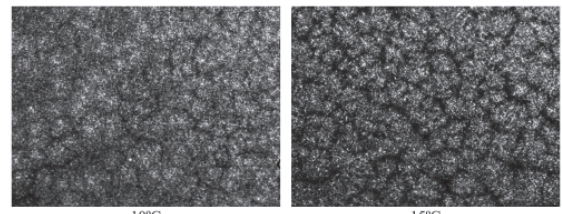

B. Interesterificada quimicamente
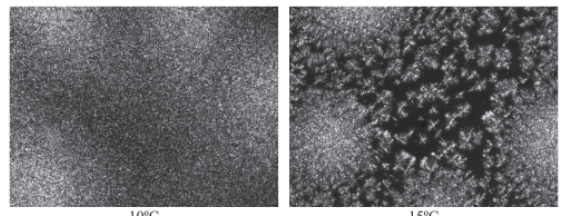

C. Interesterificada enzimaticamente
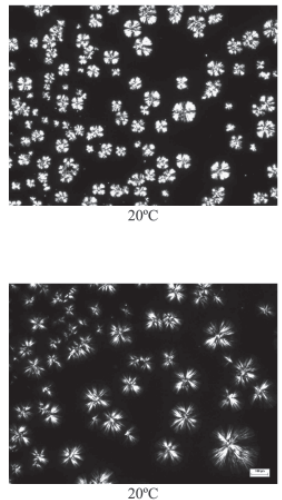

Figura 2. Imagens digitais dos cristais da amostra 2 (palmiste/peixe 80:20), em função da temperatura (a barra representa $100 \mu \mathrm{m}$ )

nos (na faixa de $5 \mu \mathrm{m}$ ) às temperaturas de 10 e $15^{\circ} \mathrm{C}$. A $20^{\circ} \mathrm{C}$, como efeito da lenta cristalização, os cristais foram muito maiores, da ordem de $200 \mu \mathrm{m}$. Após a interesterificação química e enzimática (Figuras 1B e 1C) pode-se observar que houve mudança na rede cristalina da gordura de palmiste. Esta alteração na rede cristalina do produto está provavelmente relacionada à alteração no conteúdo de gordura sólida ${ }^{22,23}$.

O óleo de peixe, antes da interesterificação, não apresentou estrutura cristalina, mesmo a $10{ }^{\circ} \mathrm{C}$. As Figuras $6 \mathrm{~A}$ e $6 \mathrm{~B}$ mostram que houve cristalização às temperaturas analisadas após as reações de interesterificação química e enzimática do óleo de peixe. Isto é de-
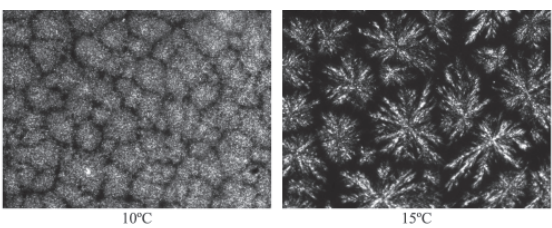

A. Não interesterificada
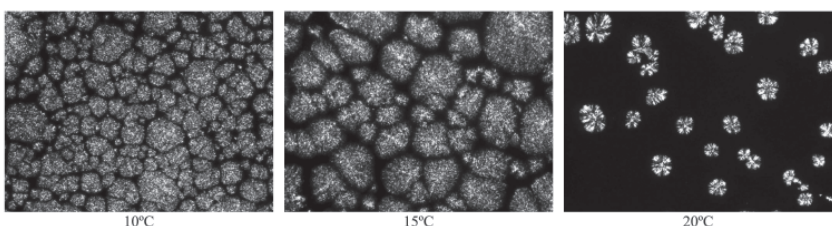

B. Interesterificada quimicamente
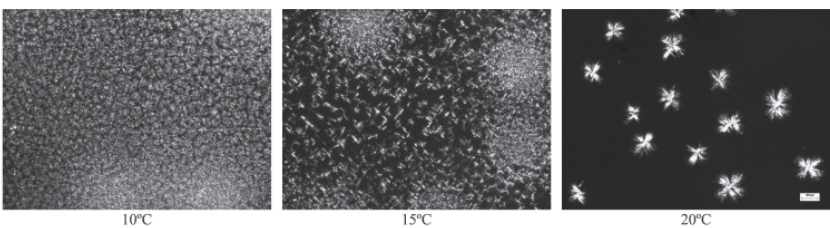

C. Interesterificada enzimaticamente

Figura 3. Imagens digitais dos cristais da amostra 3 (palmistelpeixe 60:40), em função da temperatura (a barra representa $100 \mu \mathrm{m}$ )
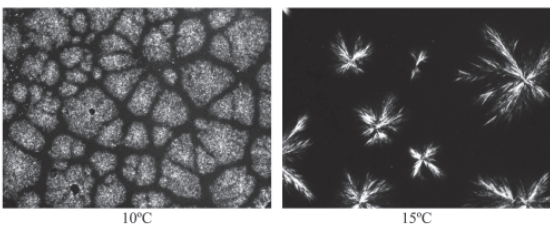

A. Não interesterificada
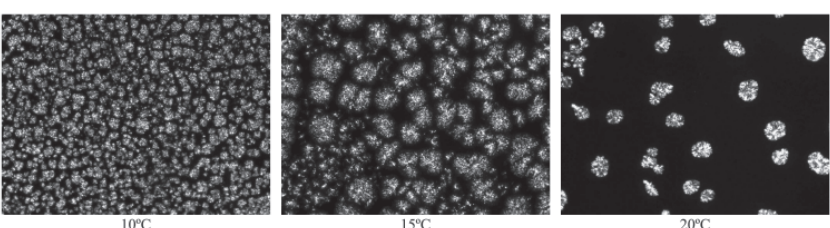

B. Interesterificada quimicamente
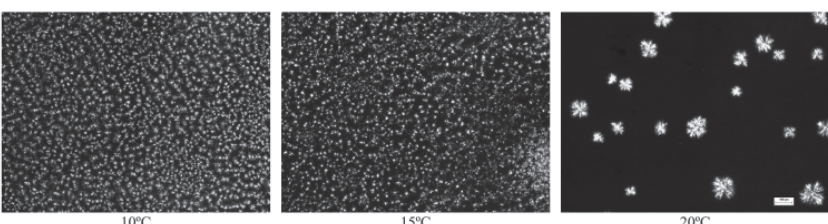

C. Interesterificada enzimaticamente

Figura 4. Imagens digitais dos cristais da amostra 4 (palmiste/peixe 40:60), em função da temperatura (a barra representa $100 \mu \mathrm{m}$ )

corrente do ponto de amolecimento ter-se elevado para $23,5^{\circ} \mathrm{C}$ após a interesterificação química e para $21,5^{\circ} \mathrm{C}$ após a interesterificação enzimática ${ }^{22,23}$. O óleo de peixe, embora seja altamente poliinsaturado, apresenta cerca de $32 \%$ de ácidos graxos saturados, principalmente representados pelos ácidos mirístico, palmítico e esteárico. Em função da interesterificação química, estes ácidos graxos são combinados aleatoriamente entre os triacilgliceróis, ocorrendo a formação de $3,2 \%$ de triacilgliceróis trissaturados (SSS) e de $20,6 \%$ de triacilgliceróis contendo dois ácidos graxos saturados e um insaturado $(\mathrm{SSI} / \mathrm{SIS} / \mathrm{ISS})^{50}$, que seriam os responsáveis pela grande elevação no ponto de amolecimento. 


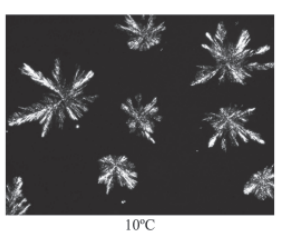

A. Não interesterificada
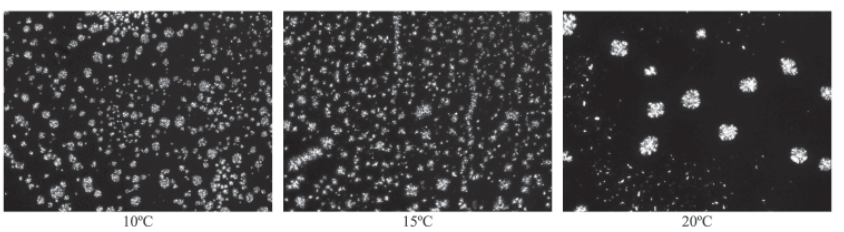

B. Interesterificada quimicament
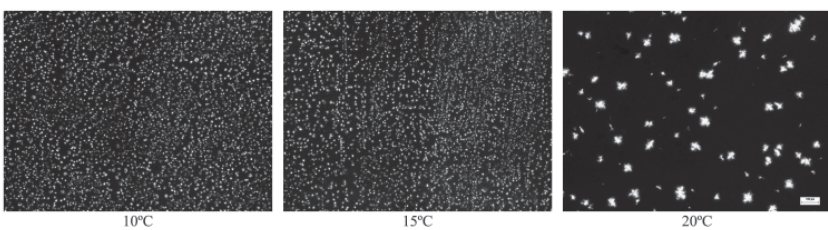

C. Interesterificada enzimaticamente

Figura 5. Imagens digitais dos cristais da amostra 5 (palmiste/peixe 20:80), em função da temperatura (a barra representa $100 \mu \mathrm{m}$ )
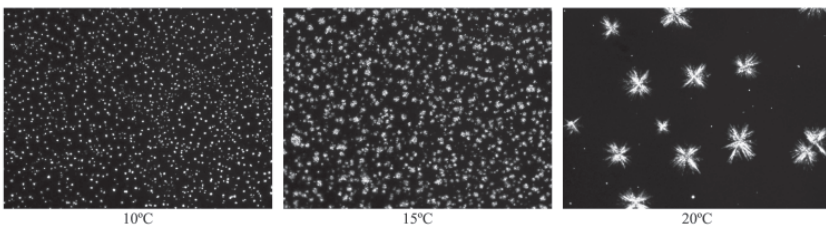

A. Interesterificada quimicamente
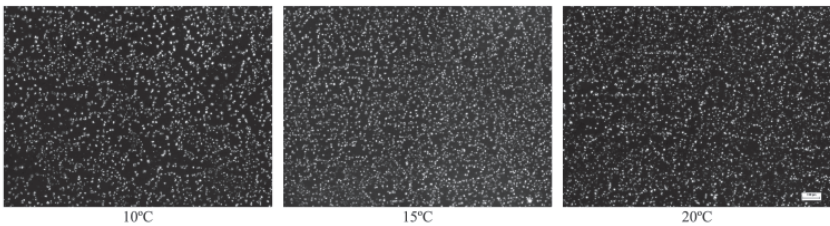

B. Interesterificada enzimaticamente

Figura 6. Imagens digitais dos cristais da amostra 6 (óleo de peixe), em função da temperatura (a barra representa $100 \mu \mathrm{m}$ )

As Figuras 2A a 5C mostram a estrutura cristalina das misturas de gordura de palmiste com óleo de peixe. Em relação à gordura de palmiste pura, as imagens mostram que a estrutura cristalina já não foi tão densa, configurando o efeito da presença do óleo de peixe na cristalização. Observa-se que se formam aglomerados de cristais (entre aproximadamente 100 e $800 \mu \mathrm{m}$ ) separados por óleo líquido, especialmente para as amostras 2 e 3 . A interesterificação promoveu aumento de cristalização dos lipídios estruturados correspondentes às amostras 4 e 5, assim como ocorreu com o conteúdo de gordura sólida ${ }^{22,23}$.

A cristalização das amostras pode ser analisada sob os pontos de vista do efeito da temperatura, da influência da presença do óleo de peixe na formação da rede cristalina e do rearranjo provocado pela interesterificação. Em relação à temperatura, para as amostras não interesterificadas os maiores cristais observados (entre aproximadamente 200 e $450 \mu \mathrm{m}$ ) foram formados à temperatura de $20^{\circ} \mathrm{C}$ para as amostras 1 e 2 , à $15^{\circ} \mathrm{C}$ para as amostras 3 e $4 \mathrm{e}$ à $10{ }^{\circ} \mathrm{C}$ para a amostra 5 . Portanto, os maiores cristais foram for- mados em temperaturas cada vez menores, à medida que aumentava a proporção de óleo de peixe na mistura. Por outro lado, para as amostras interesterificadas os maiores cristais observados (entre aproximadamente 10 e $200 \mu \mathrm{m}$ ) ocorreram geralmente à temperatura de $20^{\circ} \mathrm{C}$.

Em relação ao efeito da presença do óleo de peixe na formação da rede cristalina, os resultados indicam que a tendência de cristalização da gordura de palmiste, que é de formar cristais grandes em temperaturas alguns graus abaixo de seu ponto de fusão, se mantém nas misturas com o óleo de peixe. Por outro lado, como efeito do rearranjo nos triacilgliceróis provocado pela interesterificação, esta tendência é alterada, levando à formação de cristais menores à mesma temperatura ${ }^{54,55}$. Esta modificação na rede cristalina provocada pela interesterificação é acompanhada por alterações em propriedades físicas dos lipídios estruturados, como consistência e conteúdo de gordura sólida ${ }^{22,23}$.

Segundo Kloek et al. ${ }^{56}$ uma dispersão de cristais de gordura com grande número de cristais pequenos pode apresentar propriedades desejáveis, como boa espalhabilidade. Contudo, para fins de fracionamento, onde é necessária a separação física dos cristais, este tipo de cristalização é inadequado. O número, tamanho e tipo de cristais influenciam a propriedade de consistência de gordu$\operatorname{ras}^{57,58}$. A presença de grande número de cristais pequenos confere maior dureza à gordura que a proporcionada por poucos cristais grandes. Os cristais grandes, produzidos em resfriamento lento, conferem menor consistência à gordura, pois não formam redes interligadas. Os cristais formados por triacilgliceróis de elevado ponto de fusão oferecem maior rigidez que os de ponto de fusão mais baixo ${ }^{59}$.

Não houve muita diferença de cristalização entre os processos de interesterificação química e enzimática. Uma possível explicação para isso pode residir na migração acil em processos descontínuos catalisados por lipases, como no caso do presente trabalho.

A migração acil é um sério problema na interesterificação catalisada por lipases. A razão para esta migração é a existência de acilgliceróis parciais, especialmente diacilgliceróis, que são intermediários necessários e inevitáveis. Ela ocorre pela formação de um intermediário cíclico instável, sendo iniciada pelo ataque nucleofílico de um par de elétrons e resulta em anel intermediário de cinco membros. Este anel abre e resulta em dois produtos, o diacilglicerol original e um que apresentou migração. A migração acil da posição sn-2 para as posições sn-1 ou sn-3, ou o oposto, ocorre do mesmo modo e continua até que o equilíbrio dinâmico seja alcançado ${ }^{13,51}$. Em reatores contínuos, como o substrato entra em contato com grande quantidade de enzima, o tempo de reação é menor quando comparado com o reator descontínuo, resultando em menor migração acil. A migração acil é o principal problema em reatores descontínuos, o que resulta em menor pureza dos lipídios estruturados específicos, mesmo que a lipase seja sn-1,3 específica. A alta relação entre o substrato e a enzima exige longo tempo para a reação alcançar o equilíbrio, o que resulta conseqüentemente em migração acil. O processamento contínuo também permite a reutilização da enzima imobilizada e a redução do custo $^{52,53}$

\section{CONCLUSÕES}

Foram obtidos lipídios estruturados contendo de 4,5 a $22,8 \%$ de ácidos graxos poliinsaturados n-3 de cadeia longa e que apresentaram comportamento físico plástico, aumentando suas características de aplicação.

A adição de óleo de peixe não provocou mudanças substanci- 
ais na característica de cristalização da gordura de palmiste, que é de formação de cristais grandes em temperaturas pouco abaixo de seu ponto de fusão. Contudo, como efeito do rearranjo provocado pela interesterificação química e enzimática, foram formados cristais de menor tamanho. Os cristais observados enquadraram-se na descrição dos esferulitos dos tipos A e B.

Verificou-se que não houve muita diferença entre os processos de interesterificação química e enzimática. Uma possível explicação para isso pode residir na migração acil em processos descontínuos catalisados por lipases, como o caso do presente trabalho.

\section{AGRADECIMENTOS}

À FAPESP, CNPq e CAPES, pelo apoio financeiro e bolsas concedidas aos autores.

\section{REFERÊNCIAS}

1. Goldberg, I., ed.; Functional foods: Designer foods, pharmafoods, nutraceuticals, Chapman \& Hall: New York, 1994, p. 3

2. Willis, W. M.; Lencki, R. W.; Marangoni, A. G.; Crit. Rev. Food Sci. Nutr. 1998, 38, 639 .

3. Carvalho, P. O.; Campos, P. R. B.; D’Addio Noffs, M.; Oliveira, J. G.; Shimizu, M. T.; Silva, D. M.; Quim. Nova 2003, 26, 75.

4. Hasler, C. M.; Food Technol. 1998, 52, 63.

5. Lee, K. T.; Akoh, C. C.; Food Rev. Int. 1998, 14, 17.

6. Osborn, H. T.; Akoh, C. C.; Compr. Rev. Food Sci. Food Safety 2002, 1 , 93.

7. Gioielli, L. A. Em Entendendo a gordura: os ácidos graxos; Curi, R.; Pompéia, C.; Miyasaka, C. K.; Procópio, J., eds. Manole: São Paulo, 2002, p. 457.

8. Castro, H. F.; Mendes, A. A.; Santos, J. C.; Quim. Nova 2004, 27, 146.

9. Haumannn, B. F.; Inform 1997, 8, 1004.

10. Yamaguchi, I.; Akoh,C. C.; Lai, O. M.; J. Food Lipids 2004, 11, 65.

11. Nielsen, N. S.; Xu, X. B.; Timm-Heinrich, M.; Jacobsen, C.; J. Am. Oil Chem. Soc. 2004, 81, 375.

12. Timm-Heinrich, M.; Xu, X. B.; Nielsen, N. S.; Jacobsen, C.; Eur. Food Res. Technol. 2004, 219, 32.

13. Kim, I. H.; Ko, S. N.; Lee, S. M.; Chung, S. H.; Kim, H.; Lee, K. T.; Ha, T. Y.; J. Am. Oil Chem. Soc. 2004, 81, 537.

14. Moreno, P. A. G.; Medina, A. R.; Rubio, F. C.; Paez, B. C.; Grima, E. M.; Biotechnol. Progr. 2004, 20, 1044.

15. Jennings, B. H.; Akoh, C. C.; Food Chem. 2001, 72, 273.

16. Osorio, N. M.; Ferreira-Dias, S.; Gusmão, J. H.; J. Mol. Catal. B: Enzym. 2001, 11, 677 .

17. Akoh, C. C.; Moussata, C. O.; J. Am. Oil Chem. Soc. 2001, 78, 25.

18. Zhou, D. Q.; Xu, X. B.; Mu, L. H.; J. Food Lipids 2000, 7, 263.

19. Haraldsson, G. G.; Halldorsson, A.; Kulas, E.; J. Am. Oil Chem. Soc. 2000, 77, 1139.

20. Druschky, K.; Pscheidl, E.; Nutr. Res. 2000, 20, 1183.

21. Yankah, V. V.; Akoh, C .C.; J. Food Lipids 2000, 7, 247.

22. Díaz Gamboa, O. W.; Gioielli, L. A.; Grasas Aceites 2003a, 54, 122.

23. Díaz Gamboa, O. W.; Gioielli, L. A.; Grasas Aceites 2003b, 54, 161.
24. Sato, K.; Chem. Eng. Sci. 2001, 56, 2255.

25. Herrera, M. L.; Falabella, C.; Melgarejo, M.; Añón, M. C.; J. Am. Oil Chem. Soc. 1998, 75, 1273.

26. Gioielli, L. A.; Simões, I. S.; Rodrigues, J. N.; J. Food Eng. 2003, 57, 347.

27. Timms, R. E. Em Developments in oils and fats; Hamilton, R. J., ed.; Blackie London, 1995, p. 204

28. Marangoni, A .G.; Trends Food Sci. Technol. 2002, 13, 37.

29. Marangoni, A. G.; Hartel, R. W.; Food Technol. 1998, 52, 46

30. Wright, A. J.; Scanlon, M. G.; Hartel, R. W.; Marangoni, A. G.; J. Food Sci. 2001, 66, 1056.

31. Oliveira, M. A. L.; Sotero-Solis, V. E.; Gioielli, L. A.; Polakiewicz, B.; Tavares, M. F. M.; Electrophoresis 2003, 24, 1641.

32. Narine, S. S.; Marangoni, A. G.; Inform 1999, 10, 565.

33. Rodrigues, J. N.; Gioielli, L. A.; Food Res. Intern. 2003, 36, 149.

34. Gioielli, L. A.; Baruffaldi, R.; Rev. Farm. Bioquím. Univ. S. Paulo 1988, 24, 29.

35. Gioielli, L. A.; Baruffaldi, R.; Rev. Farm. Bioquím. Univ. S. Paulo 1987, 23,41 .

36. Gioielli, L. A.; Pitombo, R. N. M.; Vitolo, M.; Baruffaldi, R.; Oliveira, M. N.; Augusto, M. S.; J. Am. Oil Chem. Soc. 1994, 71, 579.

37. Facioli, N.L.; Gonçalves, L. A. G.; Quim. Nova 1998, 21, 16.

38. Simões, I. S.; Gioielli, L. A.; Rev. Bras. Ciênc. Farm. 1999b, 35, 259.

39. Simões, I. S.; Gioielli, L. A.; Braz. Arch. Biol. Technol. 2000, 43, 241.

40. Simões, I. S.; Gioielli, L. A.; Óleos Grãos 1999a, 8, 20.

41. Sotero-Solis, V. E.; Gioielli, L. A.; Alimentaria 2001, 38, 131.

42. Gunstone, F. D.; Harwood, J. L.; Padley, F. B.; The lipid handbook, Chapman \& Hall: London, 1994, p. 551.

43. Ong, A. S. H.; Choo, Y. M.; Ooi, C. K. Em ref. 27, p. 153.

44. Seher, A. Em Lipids: Technology; Paoletti, R.; Jacini, G.; Porcellati, R., eds.; Raven Press: New York, 1976, p. 527.

45. Berger, K. G.; Jewell, G. G.; Pollitt, R. J. M. Em Food microscopy; Vaughan, J. G., ed.; Academic Press: London, 1979, p. 445.

46. Stanley, D. W.; Aguilera, J. M.; Baker, K. W.; Jackman, R. L. Em Phase/ State transitions of foods; Rao, M. A.; Hartel, R. W., eds.; Marcel Dekker: New York, 1998, p. 1.

47. Gioielli, L. A.; Óleos Grãos 1996, 7, 21.

48. Rousseau, D.; Hill, A. R.; Marangoni, A. G.; J. Am. Oil Chem. Soc. 1996, $73,973$.

49. Rodrigues, J. N.; Anton, C.; Gioielli, L. A.; Rev. Bras. Ciênc. Farm. 2003, 39, 93.

50. Sonntag, N. O. V. Em Bailey's industrial oil and fat products; Swern, D., ed.; Wiley-Interscience: New York, 1982, p. 97.

51. Xu, X.; Balchen, S.; Hoy, C. E.; Adler-Nissen, J.; J. Am. Oil Chem. Soc. 1998, 75,301

52. Mu, H.; Xu, X.; Hoy, C. E.; J. Am. Oil Chem. Soc. 1998, 75, 1187.

53. Dalla-Vecchia, R.; Nascimento, M.G.; Soldi, V.; Quim. Nova 2004, 27, 623.

54. Grimaldi, R.; Gonçalves, L. A. G.; Gioielli, L. A.; Simões, I. S.; Grasas Aceites 2001a, 52, 349.

55. Grimaldi, R.; Gonçalves, L. A. G.; Gioielli, L. A.; Simões, I. S.; Grasas Aceites 2001b, 52, 363.

56. Kloek, W.; Walstra, P., Vliet, T.; J. Am. Oil Chem. Soc. 2000, 77, 643.

57. Larsson, K.; Lipids: Molecular organization, physical functions and technical applications, The Oil Press; Dundee, 1994, p. 7.

58. D'Agostini, D.; Ferraz, R. C.; Gioielli, L. A.; Rev. Bras. Ciênc. Farm. 2000, 36, 147

59. German, J. B.; Simoneau, C. Em Phase/State transitions of foods; Rao, M. A.; Hartel, R. W., eds.; Marcel Dekke: New York, 1998, p. 187. 